\title{
13-week subchronic toxicity study of a novel ginsenoside composition from ginseng leaves in rats
}

\author{
Won-Ho Shin ${ }^{1 \#}$, Yu Ri ${ }^{1 \#}$, Seon-Gil Do ${ }^{2}$, Young-Chul Lee ${ }^{2}$, Sang-Joon Park ${ }^{1 *}$ \\ ${ }^{1}$ Department of Histology, College of Veterinary Medicine, Kyungpook National University, Daegu, Korea \\ ${ }^{2}$ Unigen Inc., Cheonan, Korea
}

\begin{abstract}
UG0712 is a new ginsenoside extract processed from ginseng leaves. A subchronic toxicity study of UG0712 was conducted in male and female SD rats. Rats were treated with UG0712 at doses of 100, 400 and $1,600 \mathrm{mg} / \mathrm{kg} /$ day for 13 weeks, and observed followed by 4-week recovery period at a highest dose. No-treatment-related effects were observed regarding the mortality, ophthalmic examination, urinalysis and histopathology. Although the changes in clinical sign, body weight, organ weight, hematology, and serum biochemistry were observed, they were temporal and pharmacological effects. Based on the present experiment conditions, the no observed adverse effect level was considered to be more than $1,600 \mathrm{mg} / \mathrm{kg} /$ day in both sexes of rats.
\end{abstract}

Keywords: UG0712, ginsenoside extract, ginseng leaf, subchronic toxicity, SD rats

Received 8 July 2014; Revised version received 19 August 2014; Accepted 1 September 2014

Ginseng has been used as an adaptogen in traditional medicine for several thousand years [1]. Although ginseng has pharmacological effects on psychologic fuction, immune system, and diabetes, some adverse effects including nausea, diarrhea, euphoria, headaches, hypertension, hypotension, mastalgia and vaginal bleeding are also existed [2].

Pharmacologically, the main active compounds of ginseng are ginsenosides which are named as ' $\mathrm{Rx}$ ' and classified into the 20(S)-protopanaxadiol (PPD) (Rbl, $\mathrm{Rb} 2, \mathrm{Rb3}, \mathrm{Rc}, \mathrm{Rd}, \mathrm{Rg} 3, \mathrm{Rh} 2, \mathrm{Rs} 1)$ and the 20(S)protopanaxatriol (Re, Rf, Rg1, Rg2, Rh1) [3]. Ginsenosides show anti-cancer [4], anti-inflammatory [5], and neuroprotective effects [6-8]. In our previous study, we also reported that oral administration of a novel $\mathrm{Rd}$ and Rg3 extract improves exercise capacity and fatigue recovery [9].

Previous studies have reported that ginseng extract or ginsenosides are not toxic, but they used extract from root of ginseng [10] or single ginsenoside [11-13] for chronic and subchronic studies. Furthermore, ginsenoside contents and amounts of root and leaf from ginseng were quite different $[14,15]$, and one of most merit in the use of ginseng leaf is much cheaper than its root and particularly its leaf could be harvested yearly.

In this study, we have examined the potential subchronic toxicity of UG0712, a new ginsenoside extract processed from ginseng leaves ( $\mathrm{Rg} 3$ derivatives: $\sim 10 \%$ and total ginsenosides: $\sim 70 \%$ ) by repeated oral administration in Sprague-Dawley (SD) rat.

\section{Materials and Methods}

This study was conducted under Good Laboratory Practice (GLP) of the Korea Food and Drug Administration (KFDA) and performed in compliance with Testing

\footnotetext{
\#These two authors contributed equally to this work.

*Corresponding author: Sang-Joon Park, Department of Histology, College of Veterinary Medicine, Kyungpook National University, 1370 Sankyuk-dong, Bukgu, Daegu, 702-701, Korea

Tel: +82-53-950-5970; Fax: +82-53-950-5955; E-mail: psj26@knu.ac.kr
}

This is an Open Access article distributed under the terms of the Creative Commons Attribution Non-Commercial License (http://creativecommons.org/licenses/ by-nc/3.0) which permits unrestricted non-commercial use, distribution, and reproduction in any medium, provided the original work is properly cited. 
Guidelines for Safety Evaluation of Drugs (Notification No. 2000-63 issued by the KFDA).

\section{Preparation of UG0712}

UG0712 was prepared by blending the partially hydrolyzed with ginseng leaf extract to meet constant content of ginsenosides $\mathrm{Rd}$ and $\mathrm{Rg} 3$. Briefly, ginseng leaf extract which was purchased from Hongju Biotech Co., Ltd (Yanbian, China) was easily hydrolyzed under acidic condition. UG0712 was standardized with above $5 \%$ of ginsenoside $\mathrm{Rd}$, above $10 \%$ of ginsenoside $\mathrm{Rg} 3$ derivatives ( $\mathrm{Rg} 3, \mathrm{Rg} 5$ and $\mathrm{Rk} 1)$.

\section{High performance liquid chromatography (HPLC) analysis of UG0712}

For analyzing freeze-dried ginseng extracts, it was dissolved in methanol with $2 \mathrm{mg} / \mathrm{mL}$ concentration, respectively. HPLC analysis was conducted on a HITACHI HPLC system equipped with L-7455 detector. A capcell PAK C18 column $(5 \mu \mathrm{m}, 3.0 \mathrm{~mm} * 75 \mathrm{~mm})$ was used and maintained at $40^{\circ} \mathrm{C}$. A gradient solvent composition of acetonitrile and deionized water was used. The flow rate and injection volume were $0.5 \mathrm{~mL} /$ min and $10 \mu \mathrm{L}$, respectively. The chromatograms were obtained at a wavelength of $203 \mathrm{~nm}$ with UV detector. Standard sample of ginsenoside was prepared with 0.2 $\mathrm{mg} / \mathrm{mL}$ concentration.

\section{Test substance and formulation}

UG0712, an extract powder processed from ginseng leaves (Lot No. UG0712-BL05002), was supplied by Unigen, Inc (Cheonan, Chugnam, Korea). For administration, the test substance was suspended into the vehicle (Corn oil, Sigma-Aldrich Inc., MO, USA) and dosing formulations were prepared daily.

\section{Animals}

Male and female 5-6 weeks old SD rats were obtained from the Orientbio Inc. (Sungnam, Gyeonggi, Korea). The body weight range on receipt ranged from 112.8 to $137.7 \mathrm{~g}$ for males and 91.1 to $120.4 \mathrm{~g}$ for female. The animals were used after 6 day acclimatization and housed one animal to a stainless wire cage in a room with a barrier system controlled for the light-dark cycle (12-12 h), air exchange (10-15 changes/h), and temperature $\left(19.8-24.7^{\circ} \mathrm{C}\right)$ during the study.

\section{Feed and water}

Animals were provided Teklad cerified global 18\% protein rodent diet (Harlan Teklad, WI, USA) and filtered municipal tap water. Food and water were provided ad libitum.

\section{Administration and study design}

At the end of the quarantine and acclimation period, animals with findings in clinical signs and abnormalities in body weight increment were eliminated from the randomization pool. One-hundred twelve animals (56 males and 56 females) were randomly divided into four groups and administered test substance at doses of 0 (vehicle), 100, 400, 1,600 mg/kg. The dosing volume was $5 \mathrm{~mL} / \mathrm{kg}$. Test substance and vehicle were orally administered inside the stomach using a sonde attached by a disposable syringe once a day. The repeated dose toxicity included both a 13-week treatment period for all dosing groups (main groups) and a 4-week recovery period for 0 and $1,600 \mathrm{mg} / \mathrm{kg}$ dosing groups (recovery groups). During the treatment period, all animals were observed for clinical signs at least once daily. Body weights and food consumptions were measured just before dosing, once weekly for 13 weeks and the day of necropsy.

\section{Ophthalmic examination and urinalysis}

Ophthalmic evaluation was conducted once prior to the necropsy through macroscopic and ophthalmoscopic examinations (PRACTITIONER, Keeler, UK).

The urine samples were collected for urinalysis from animals prior to the necropsy. The urinalysis was performed using urine analyzer (MIDITRON Junior II, Roche, Germany) and stick (Combur ${ }^{10}$ Test $^{\circledR} \mathrm{M}$, Roche, Germany) to evaluate the following parameters; color, transparency, volume, specific gravity, $\mathrm{pH}$, protein, glucose, ketone, bilirubin, and erythrocyte. The sediment test was also performed microscopically.

\section{Hematology and serum biochemistry}

Hematological and serum biochemical determinations were performed from samples collected from an abdominal artery. Hematology samples were taken into tubes containing ethylenediamine tetraacetic acid (EDTA) and analyzed using the hematological autoanalyzer (CELLDYN 3700, Abbott, USA). Parameters including total 
red blood cell count (RBC), hemoglobin concentration (HGB), hematocrit (HCT), RBC indices, total white blood cell count (WBC), platelet (PLT), WBC differential count, and reticulocyte (Retic). The blood smear specimens were prepared and stained with Diff-Quick stain (Hemacolor ${ }^{\circledR}$, Merk, Germany) for the calculation of the leukocyte percentage and new methylene blue dye for reticulocyte calculation. In addition, blood samples treated with $3.2 \%$ sodium citrate were analyzed for prothrombin time (PT) and activated partial thromboplastin time (APTT) by the automatic coagulation time meter (ACL 7000, Instrumental Laboratory, USA).

Serum biochemistry measurements of the following parameters were performed by INTEGRA 400 (Roche, Germany) and AVL9181 (Roche, Germany): Alanine aminotransferase (ALT), aspartate aminotransferase (AST), alkaline phosphatase (ALP), $\gamma$-gluramyltranspeptidase $(\gamma$-GT), blood urea nitrogen (BUN), creatinine (Creat), total bilirubin (T-Bili), total protein (TP), albumin (Alb), albumin/globulin ratio (A/G), total cholesterol (T-Chol), triglycerides (TG), phosphorus (P), glucose (Glu), calcium $(\mathrm{Ca})$, chloride $(\mathrm{Cl})$, sodium $(\mathrm{Na})$, and potassium $(\mathrm{K})$.

\section{Gross observation and organ weights}

All animals were exsanguinated after the blood collection, recorded gross findings about full external surface, cranial cavity, and organs of thoracic and abdominal cavities. The absolute and relative (organ-tobody weight ratios) weights of organs including liver, heart, brain, kidneys, testes, ovaries, spleen, lungs, pituitary, adrenals, prostate, and uterus were measured.

\section{Histopathology}

Following tissues were collected and preserved at the
$10 \%$ neutral buffer formalin except for testes which were fixed in the Davidson fixative: Liver, trachea, kidneys, spleen, esophagus, duodenum, ileum, colon, pancreas, brain, thyroids, testes, prostate, ovaries, lung, heart, adrenals, thymus, stomach, jejunum, cecum, rectum, submandibular gland, pituitary, urinary bladder, epididymides, seminal vesicle, and uterus. Fixed tissues were embedded in paraffin to make $3 \mu \mathrm{m}$ tissue sectioned and stained with Hematoxylin and Eosin. Histopathological assessment was performed on all tissues of the control and highest dose group animals microscopically.

\section{Statistical analysis}

For the data including body weight, food consumption, urine volume, hematology and serum biochemisty and organ weight parameters, the Levene's test was conducted to test for variance of homogeneity. In the case of heterogeneity, proper data transformation was used to stabilize the variance. One-way analysis of variance (ANOVA) was employed on homogeneous or transformed data and then, if significant, followed by Dunnett's test for multiple comparisons.

\section{Results}

\section{HPLC anaylsis of UG0712}

Ginsenosides, such as Rb1-3, Rc, Rd, Re, Rg1, Rg2, $\mathrm{Rg} 3, \operatorname{Rg} 5$ and Rk1 were analyzed. The HPLC profile revealed a high concentration of $\operatorname{Rg} 3, \operatorname{Rg} 5$ and $\mathrm{Rk} 1$ in comparison to the other ginsenosides. The contents of each ginsenosides in total in ginseng leaves extract are Rb1 (0.8\%), Rb2 (1.6\%), Rb3 (0.5\%), Rc (1.1\%), Rd (7.8\%), Re (5.7), Rg1 (2.1\%), Rg2 (12.1\%), Rg3 (6\%), $\operatorname{Rg} 5$ (2.4\%) and Rk1 (1.8\%), respectively. Also we show

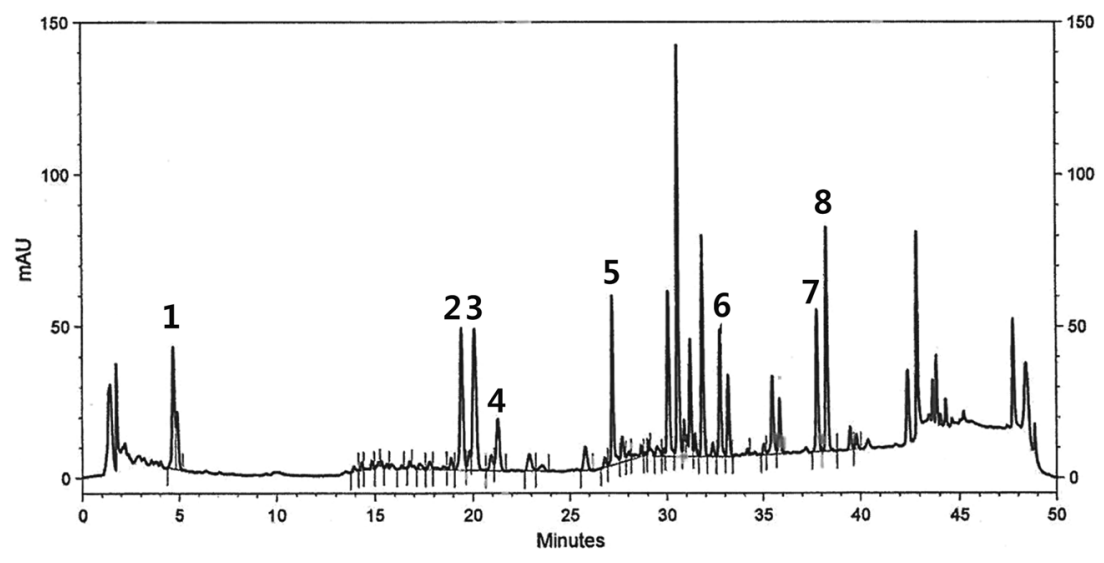

Figure1. Representative HPLC profile of the UG0712. $\operatorname{Rg} 1(1), \operatorname{Rb} 1(2), \operatorname{Rc}(3), \operatorname{Rb} 2(4), \operatorname{Rd}(5), \operatorname{Rg} 3(6), \operatorname{Rk} 1(7)$ and $\operatorname{Rg} 5(8)$. 
Table 1. Body weight changes of male and female rats treated with UG0712

\begin{tabular}{|c|c|c|c|c|}
\hline Study weeks & Vehicle control & $100 \mathrm{mg} / \mathrm{kg}$ & $400 \mathrm{mg} / \mathrm{kg}$ & $1,600 \mathrm{mg} / \mathrm{kg}$ \\
\hline \multicolumn{5}{|l|}{ Males } \\
\hline No. of rats & 17 & 11 & 11 & 17 \\
\hline 0 & $176.95 \pm 6.72$ & $177.80 \pm 6.53$ & $178.61 \pm 6.43$ & $178.21 \pm 5.41$ \\
\hline 1 & $240.20 \pm 13.45$ & $242.24 \pm 11.11$ & $241.41 \pm 9.15$ & $241.67 \pm 7.78$ \\
\hline 2 & $300.86 \pm 17.99$ & $301.18 \pm 13.33$ & $303.84 \pm 14.71$ & $301.20 \pm 12.99$ \\
\hline 3 & $358.21 \pm 21.46$ & $358.97 \pm 19.93$ & $363.22 \pm 20.76$ & $358.09 \pm 18.88$ \\
\hline 4 & $399.90 \pm 26.42$ & $398.04 \pm 23.51$ & $410.57 \pm 25.36$ & $402.18 \pm 23.79$ \\
\hline 5 & $434.53 \pm 31.79$ & $430.99 \pm 26.63$ & $448.70 \pm 31.55$ & $444.03 \pm 28.37$ \\
\hline 6 & $468.34 \pm 34.15$ & $464.54 \pm 28.90$ & $484.86 \pm 37.19$ & $477.02 \pm 31.72$ \\
\hline 7 & $498.08 \pm 37.30$ & $494.27 \pm 33.06$ & $514.87 \pm 40.35$ & $505.63 \pm 35.09$ \\
\hline 8 & $524.61 \pm 40.45$ & $517.74 \pm 34.04$ & $544.83 \pm 43.53$ & $533.05 \pm 37.58$ \\
\hline 9 & $548.04 \pm 42.24$ & $538.70 \pm 38.38$ & $571.33 \pm 45.73$ & $556.62 \pm 41.26$ \\
\hline 10 & $565.21 \pm 44.68$ & $558.11 \pm 41.60$ & $592.37 \pm 46.41$ & $570.95 \pm 38.92$ \\
\hline 11 & $583.41 \pm 47.96$ & $576.20 \pm 42.59$ & $614.47 \pm 46.82$ & $588.96 \pm 44.68$ \\
\hline 12 & $600.80 \pm 49.72$ & $592.48 \pm 46.16$ & $633.76 \pm 48.50$ & $606.22 \pm 46.78$ \\
\hline 13 & $608.84 \pm 44.64$ & $604.27 \pm 50.10$ & $648.44 \pm 50.56$ & $618.49 \pm 48.13$ \\
\hline \multicolumn{5}{|l|}{ Recovery period } \\
\hline No. of rats & 6 & - & - & 6 \\
\hline 14 & $596.21 \pm 37.23$ & - & - & $631.21 \pm 38.86$ \\
\hline 15 & $605.31 \pm 38.73$ & - & - & $644.68 \pm 42.12$ \\
\hline 16 & $615.00 \pm 41.74$ & - & - & $653.53 \pm 41.95$ \\
\hline 17 & $619.65 \pm 40.89$ & - & - & $658.69 \pm 45.54$ \\
\hline \multicolumn{5}{|l|}{ Females } \\
\hline \multicolumn{5}{|l|}{ Treatment period } \\
\hline No. of rats & 17 & 11 & 11 & 17 \\
\hline 0 & $148.11 \pm 6.95$ & $148.44 \pm 6.64$ & $147.88 \pm 7.41$ & $146.81 \pm 7.29$ \\
\hline 1 & $176.18 \pm 10.38$ & $179.53 \pm 8.73$ & $180.30 \pm 9.90$ & $179.68 \pm 10.02$ \\
\hline 2 & $196.17 \pm 15.03$ & $201.83 \pm 10.95$ & $201.31 \pm 11.17$ & $203.00 \pm 13.54$ \\
\hline 3 & $218.56 \pm 16.00$ & $227.59 \pm 13.22$ & $228.73 \pm 12.19$ & $231.09 \pm 16.03$ \\
\hline 4 & $230.98 \pm 21.36$ & $242.84 \pm 16.46$ & $245.19 \pm 11.03$ & $246.89 \pm 18.81$ \\
\hline 5 & $245.39 \pm 25.04$ & $257.58 \pm 17.36$ & $263.76 \pm 13.30$ & $262.91 \pm 18.32 *$ \\
\hline 6 & $258.67 \pm 27.18$ & $272.40 \pm 19.83$ & $279.39 \pm 14.61$ & $278.13 \pm 19.20^{*}$ \\
\hline 7 & $269.20 \pm 26.78$ & $284.02 \pm 19.30$ & $288.97 \pm 16.85$ & $288.05 \pm 20.19^{*}$ \\
\hline 8 & $277.52 \pm 30.43$ & $292.34 \pm 19.47$ & $297.39 \pm 17.79$ & $295.56 \pm 22.34$ \\
\hline 9 & $286.38 \pm 32.65$ & $299.41 \pm 21.76$ & $306.88 \pm 19.23$ & $304.00 \pm 20.51$ \\
\hline 10 & $294.30 \pm 32.49$ & $308.76 \pm 23.55$ & $315.99 \pm 20.33$ & $311.34 \pm 23.80$ \\
\hline 11 & $300.43 \pm 33.42$ & $316.03 \pm 25.27$ & $325.44 \pm 25.09$ & $318.94 \pm 22.61$ \\
\hline 12 & $307.36 \pm 35.28$ & $326.21 \pm 25.79$ & $332.05 \pm 27.13$ & $325.88 \pm 25.06$ \\
\hline 13 & $314.54 \pm 36.25$ & $330.27 \pm 27.07$ & $335.68 \pm 26.06$ & $330.09 \pm 26.16$ \\
\hline \multicolumn{5}{|l|}{ Recovery period } \\
\hline No. of rats & 6 & - & - & 6 \\
\hline 14 & $315.09 \pm 10.42$ & - & - & $327.28 \pm 35.35$ \\
\hline 15 & $319.73 \pm 15.00$ & - & - & $333.87 \pm 38.52$ \\
\hline 16 & $321.16 \pm 17.07$ & - & - & $333.73 \pm 38.38$ \\
\hline 17 & $333.59 \pm 15.72$ & - & - & $339.95 \pm 41.23$ \\
\hline
\end{tabular}

Values are expressed as means(g) \pm SD. ${ }^{*} P<0.05$ vs vehicle control.

the corresponding chromatogram in Figure 1.

\section{Mortality, clinical sign, and body weight}

No mortality related to UG0712 administration was observed in this study. Salivation was seen in all UG0712treated animals during a 13 -week treatment period. Soft stool, diarrhea and mucous stool were also showed sporadically in animals of both genders treated with 400 and 1,600 mg/kg UG0712 during a 13-week treatment period. However, these clinical signs were not observed in a recovery period.

There were no significantly differences in body weight 
Table 2. Hematological parameters in male and female rats treated with UG0712

\begin{tabular}{|c|c|c|c|c|}
\hline Parameters & Vehicle control & $100 \mathrm{mg} / \mathrm{kg}$ & $400 \mathrm{mg} / \mathrm{kg}$ & $1,600 \mathrm{mg} / \mathrm{kg}$ \\
\hline \multicolumn{5}{|l|}{ Males } \\
\hline No. of rats & 11 & 11 & 11 & 11 \\
\hline $\operatorname{RBC}\left(x 10^{6} / \mu \mathrm{L}\right)$ & $7.81 \pm 0.27$ & $7.92 \pm 0.26$ & $7.74 \pm 0.3$ & $7.97 \pm 0.33$ \\
\hline HGB (g/dL) & $14.0 \pm 0.5$ & $14.1 \pm 0.5$ & $13.7 \pm 0.5$ & $14.1 \pm 0.3$ \\
\hline HCT (\%) & $41.7 \pm 1.3$ & $41.8 \pm 1.1$ & $40.8 \pm 1.8$ & $42.3 \pm 1.5$ \\
\hline Reti (\%) & $2.12 \pm 0.42$ & $1.88 \pm 0.49$ & $1.81 \pm 0.36$ & $1.97 \pm 0.47$ \\
\hline MCV (fL) & $53.4 \pm 1.8$ & $52.8 \pm 1.4$ & $52.8 \pm 1.9$ & $53.1 \pm 1.6$ \\
\hline $\mathrm{MCH}(\mathrm{pg})$ & $18.0 \pm 0.7$ & $17.8 \pm 0.6$ & $17.7 \pm 0.5$ & $17.8 \pm 0.8$ \\
\hline $\mathrm{MCHC}(\mathrm{g} / \mathrm{dL})$ & $33.7 \pm 0.8$ & $33.7 \pm 0.7$ & $33.7 \pm 0.9$ & $33.5 \pm 1.0$ \\
\hline 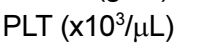 & $1007 \pm 95$ & $1040 \pm 70$ & $1067 \pm 108$ & $1066 \pm 110$ \\
\hline WBC $\left(x 10^{3} / \mu \mathrm{L}\right)$ & $11.66 \pm 2.30$ & $10.83 \pm 2.23$ & $11.31 \pm 3.15$ & $11.75 \pm 3.00$ \\
\hline NEU (\%) & $20.4 \pm 5.9$ & $18.0 \pm 5.1$ & $21.3 \pm 7.7$ & $21.8 \pm 5.1$ \\
\hline LYM (\%) & $74.1 \pm 7.1$ & $76.6 \pm 4.9$ & $72.3 \pm 8.2$ & $73.9 \pm 6.2$ \\
\hline MONO (\%) & $1.4 \pm 1.2$ & $1.4 \pm 0.8$ & $1.7 \pm 0.8$ & $0.8 \pm 0.7$ \\
\hline EOS (\%) & $1.4 \pm 0.4$ & $1.0 \pm 0.3$ & $1.3 \pm 0.5$ & $1.1 \pm 0.3$ \\
\hline BASO (\%) & $2.7 \pm 1.3$ & $3.0 \pm 1.1$ & $3.4 \pm 1.3$ & $2.5 \pm 1.3$ \\
\hline PT (sec) & $16.3 \pm 0.8$ & $15.7 \pm 0.4$ & $15.9 \pm 0.8$ & $15.8 \pm 0.5$ \\
\hline APTT (sec) & $22.6 \pm 1.8$ & $22.2 \pm 1.4$ & $22.3 \pm 2.4$ & $22.6 \pm 1.7$ \\
\hline $\begin{array}{l}\text { Recovery group } \\
\text { No. of rats }\end{array}$ & 6 & - & 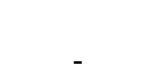 & 6 \\
\hline $\mathrm{RBC}\left(\mathrm{x} 10^{6} / \mu \mathrm{L}\right)$ & $7.84 \pm 0.23$ & - & - & $7.76 \pm 0.20$ \\
\hline HGB (g/dL) & $13.7 \pm 0.7$ & - & - & $13.8 \pm 0.4$ \\
\hline HCT (\%) & $40.9 \pm 1.9$ & - & - & $41.1 \pm 1.3$ \\
\hline Reti (\%) & $3.40 \pm 0.59$ & - & - & $2.77 \pm 0.59$ \\
\hline MCV (fL) & $52.1 \pm 1.8$ & - & - & $53.0 \pm 2.0$ \\
\hline $\mathrm{MCH}(p g)$ & $17.4 \pm 0.8$ & - & - & $17.8 \pm 0.8$ \\
\hline $\mathrm{MCHC}(\mathrm{g} / \mathrm{dL})$ & $33.4 \pm 0.5$ & - & - & $33.5 \pm 0.4$ \\
\hline $\operatorname{PLT}\left(x 10^{3} / \mu \mathrm{L}\right)$ & $1076 \pm 78$ & - & - & $1105 \pm 95$ \\
\hline WBC $\left(x 10^{3} / \mu \mathrm{L}\right)$ & $8.94 \pm 2.62$ & - & - & $10.77 \pm 2.89$ \\
\hline NEU (\%) & $21.2 \pm 9.0$ & - & - & $20.0 \pm 6.7$ \\
\hline LYM (\%) & $73.3 \pm 9.6$ & - & - & $71.6 \pm 8.2$ \\
\hline MONO (\%) & $1.2 \pm 1.2$ & - & - & $2.2 \pm 1.2$ \\
\hline EOS (\%) & $2.4 \pm 0.4$ & - & - & $2.8 \pm 1.0$ \\
\hline BASO (\%) & $2.0 \pm 1.9$ & - & - & $3.5 \pm 1.8$ \\
\hline PT (sec) & $16.0 \pm 0.4$ & - & - & $17.1 \pm 1.1^{*}$ \\
\hline APTT (sec) & $21.6 \pm 2.0$ & - & - & $21.2 \pm 0.9$ \\
\hline
\end{tabular}

between vehicle control and UG0712-treated male rats, but a tendency for its increase was observed. However, body weights were increased at $1,600 \mathrm{mg} / \mathrm{kg}$ from week 5 to week 7 in females (Table 1). Food consumption was increased at week 5, 8, 12, 13 and 14 in male and at week 3 and 4 in female treated with $1,600 \mathrm{mg} / \mathrm{kg}$ UG0712 when compared with the vehicle control group (data not shown).

\section{Ophthalmic examination and urinalysis}

There were no ocular changes attributed to the treatment of test substance and no treatment related changes in urinalysis parameter in any treatment groups (data not shown).

\section{Hematology and serum biochemistry}

The results of hematology examination are shown in Table 2. After the 13-week treatment, there were no significant treatment related alterations noted in all animals. However, prothrombin time was significantly increased in male recovery group at $1,600 \mathrm{mg} / \mathrm{kg}$. In females, reticulocyte and eosinophil were decreased, but platelet was increased in the $1,600 \mathrm{mg} / \mathrm{kg}$ recovery group.

\section{Gross observation and organ weights}

No treatment related gross findings were shown in any of the treated animals. Absolute and relative organ weights are shown in Table 4. There were no differences 
Table 2. Continued

\begin{tabular}{|c|c|c|c|c|}
\hline Parameters & Vehicle control & $100 \mathrm{mg} / \mathrm{kg}$ & $400 \mathrm{mg} / \mathrm{kg}$ & $1,600 \mathrm{mg} / \mathrm{kg}$ \\
\hline \multicolumn{5}{|l|}{$\begin{array}{l}\text { Females } \\
\text { Main group }\end{array}$} \\
\hline No. of rats & 11 & 11 & 11 & 11 \\
\hline $\operatorname{RBC}\left(x 10^{6} / \mu \mathrm{L}\right)$ & $7.30 \pm 0.27$ & $7.29 \pm 0.29$ & $7.16 \pm 0.44$ & $7.25 \pm 0.33$ \\
\hline HGB (g/dL) & $13.5 \pm 0.5$ & $13.6 \pm 0.6$ & $13.2 \pm 0.6$ & $13.5 \pm 0.5$ \\
\hline HCT (\%) & $40.5 \pm 1.7$ & $40.9 \pm 1.8$ & $39.8 \pm 1.6$ & $40.7 \pm 1.6$ \\
\hline Reti (\%) & $1.60 \pm 0.25$ & $1.92 \pm 0.64$ & $1.83 \pm 0.45$ & $1.76 \pm 0.43$ \\
\hline MCV (fL) & $55.5 \pm 1.4$ & $56.1 \pm 1.6$ & $55.6 \pm 1.8$ & $56.1 \pm 1.0$ \\
\hline $\mathrm{MCH}(\mathrm{pg})$ & $18.6 \pm 0.4$ & $18.6 \pm 0.6$ & $18.5 \pm 0.6$ & $18.6 \pm 0.3$ \\
\hline $\mathrm{MCHC}(\mathrm{g} / \mathrm{dL})$ & $33.4 \pm 0.7$ & $33.3 \pm 0.7$ & $33.3 \pm 0.2$ & $33.2 \pm 0.5$ \\
\hline $\operatorname{PLT}\left(x 10^{3} / \mu \mathrm{L}\right)$ & $1032 \pm 100$ & $1024 \pm 98$ & $1029 \pm 127$ & $1054 \pm 77$ \\
\hline WBC $\left(x 10^{3} / \mu \mathrm{L}\right)$ & $5.57 \pm 2.12$ & $4.91 \pm 1.72$ & $5.10 \pm 1.68$ & $5.14 \pm 1.28$ \\
\hline NEU (\%) & $19.6 \pm 7.4$ & $16.1 \pm 3.8$ & $18.4 \pm 8.7$ & $21.5 \pm 6.7$ \\
\hline LYM (\%) & $75.9 \pm 7.8$ & $79.5 \pm 5.0$ & $77.0 \pm 8.6$ & $74.6 \pm 6.3$ \\
\hline MONO (\%) & $1.0 \pm 0.5$ & $1.0 \pm 0.7$ & $1.1 \pm 0.9$ & $1.0 \pm 0.7$ \\
\hline EOS (\%) & $1.2 \pm 0.5$ & $1.4 \pm 0.5$ & $1.2 \pm 0.3$ & $1.1 \pm 0.2$ \\
\hline BASO (\%) & $2.2 \pm 1.2$ & $2.1 \pm 0.8$ & $2.3 \pm 1.2$ & $1.9 \pm 1.2$ \\
\hline PT (sec) & $15.1 \pm 1.3$ & $15.5 \pm 0.5$ & $15.3 \pm 0.6$ & $15.0 \pm 0.8$ \\
\hline APTT (sec) & $19.2 \pm 1.7$ & $19.9 \pm 2.5$ & $19.7 \pm 2.0$ & $20.0 \pm 1.9$ \\
\hline $\begin{array}{l}\text { Recovery group } \\
\text { No. of rats }\end{array}$ & 6 & - & 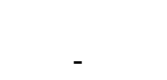 & 6 \\
\hline $\operatorname{RBC}\left(x 10^{6} / \mu \mathrm{L}\right)$ & $7.22 \pm 0.32$ & - & - & $7.27 \pm 0.40$ \\
\hline HGB (g/dL) & $13.4 \pm 0.8$ & - & - & $13.6 \pm 0.6$ \\
\hline HCT (\%) & $40.1 \pm 1.9$ & - & - & $40.6 \pm 2.2$ \\
\hline Reti (\%) & $2.82 \pm 0.25$ & - & - & $1.97 \pm 0.30^{* \star *}$ \\
\hline MCV (fL) & $55.6 \pm 1.1$ & - & - & $55.8 \pm 1.4$ \\
\hline $\mathrm{MCH}(p g)$ & $18.6 \pm 0.4$ & - & - & $18.7 \pm 0.5$ \\
\hline $\mathrm{MCHC}(\mathrm{g} / \mathrm{dL})$ & $33.5 \pm 0.5$ & - & - & $33.6 \pm 0.5$ \\
\hline $\operatorname{PLT}\left(x 10^{3} / \mu \mathrm{L}\right)$ & $889 \pm 65$ & - & - & $1018 \pm 55^{\star *}$ \\
\hline WBC $\left(x 10^{3} / \mu \mathrm{L}\right)$ & $3.09 \pm 0.34$ & - & - & $4.58 \pm 2.08$ \\
\hline NEU $(\%)$ & $22.1 \pm 12.6$ & - & - & $22.1 \pm 5.40$ \\
\hline LYM (\%) & $70.3 \pm 13.2$ & - & - & $72.3 \pm 5.10$ \\
\hline MONO (\%) & $1.7 \pm 1.0$ & - & - & $1.5 \pm 0.4$ \\
\hline EOS (\%) & $2.2 \pm 1.0$ & - & - & $1.2 \pm 0.3^{*}$ \\
\hline BASO (\%) & $3.8 \pm 1.7$ & - & - & $3.0 \pm 1.0$ \\
\hline PT (sec) & $16.0 \pm 0.4$ & - & - & $16.0 \pm 0.8$ \\
\hline APTT (sec) & $21.4 \pm 2.7$ & - & - & $21.7 \pm 3.7$ \\
\hline
\end{tabular}

Values are expressed as means \pm SD. ${ }^{*} P<0.05$, ${ }^{\star *} P<0.01$, and ${ }^{* \star *} P<0.001$ vs vehicle control.

Serum tests (Table 3) revealed that chloride was increased at $1600 \mathrm{mg} / \mathrm{kg}$ in males on week 13 . In females at 400 and $1600 \mathrm{mg} /$ $\mathrm{kg}$, total protein was increased after 13-week treatment. After the 4-week recovery period, glucose and potassium were decreased in females at $1600 \mathrm{mg} / \mathrm{kg}$.

in absolute organ weight in males of main and recovery groups, while decrease in the relative weights of the testes in the $400 \mathrm{mg} / \mathrm{kg}$ main group and the pituitary in the $1,600 \mathrm{mg} / \mathrm{kg}$ recovery group were apparent. In the female, increase of the absolute and relative liver weights was observed in the 400 and $1,600 \mathrm{mg} / \mathrm{kg}$ main groups, but there was no significant difference in all organ weights between vehicle control and treated animals in recovery groups.

\section{Histopathological findings}

The results of histopathological examination did not show any abnormal changes in all animals (data not shown).

\section{Discussion}

This study was conducted to assess the cumulative toxicity of UG0712, a new ginsenoside extract processed 
Table 3. Serum biochemical parameters in male and female rats treated with UG0712

\begin{tabular}{|c|c|c|c|c|}
\hline Parameters & Vehicle control & 100 mg/kg & 400 mg/kg & $1,600 \mathrm{mg} / \mathrm{kg}$ \\
\hline \multicolumn{5}{|l|}{ Males } \\
\hline No. of rats & 11 & 11 & 11 & 11 \\
\hline ALT (U/L) & $41.4 \pm 20.2$ & $33.6 \pm 9.7$ & $30.0 \pm 5.9$ & $29.2 \pm 4.7$ \\
\hline AST (U/L) & $86.1 \pm 43.2$ & $92.5 \pm 33.9$ & $81.2 \pm 18.5$ & $84.0 \pm 21.0$ \\
\hline ALP (U/L) & $89.4 \pm 28.1$ & $93.5 \pm 27.4$ & $83.0 \pm 12.8$ & $91.0 \pm 17.6$ \\
\hline Glu (mg/dL) & $134.0 \pm 11.2$ & $130.0 \pm 12.9$ & $132.6 \pm 13.1$ & $126.6 \pm 13.2$ \\
\hline BUN (mg/dL) & $9.16 \pm 0.92$ & $9.98 \pm 1.08$ & $10.12 \pm 1.22$ & $10.53 \pm 1.62$ \\
\hline Crea (mg/dL) & $0.33 \pm 0.05$ & $0.32 \pm 0.04$ & $0.34 \pm 0.05$ & $0.36 \pm 0.05$ \\
\hline T-Bil (mg/dL) & $0.17 \pm 0.1$ & $0.14 \pm 0.05$ & $0.15 \pm 0.08$ & $0.10 \pm 0.02$ \\
\hline T-Chol (mg/dL) & $75.55 \pm 14.49$ & $71.92 \pm 21.64$ & $82.53 \pm 15.28$ & $72.87 \pm 12.97$ \\
\hline TG (mg/dL) & $66.53 \pm 20.77$ & $55.53 \pm 24.22$ & $67.12 \pm 18.29$ & $58.44 \pm 38.59$ \\
\hline $\mathrm{TP}(\mathrm{g} / \mathrm{dL})$ & $6.6 \pm 0.2$ & $6.7 \pm 0.2$ & $6.6 \pm 0.2$ & $6.4 \pm 0.6$ \\
\hline Alb (g/dL) & $4.0 \pm 0.1$ & $4.1 \pm 0.2$ & $4.0 \pm 0.2$ & $4.1 \pm 0.3$ \\
\hline A/G ratio & $1.56 \pm 0.12$ & $1.64 \pm 0.18$ & $1.54 \pm 0.2$ & $1.98 \pm 0.81$ \\
\hline$P(\mathrm{mg} / \mathrm{dL})$ & $6.95 \pm 0.39$ & $7.26 \pm 0.43$ & $7.55 \pm 0.61$ & $7.56 \pm 0.82$ \\
\hline $\mathrm{Ca}(\mathrm{mmol} / \mathrm{L})$ & $2.65 \pm 0.09$ & $2.64 \pm 0.06$ & $2.65 \pm 0.08$ & $2.66 \pm 0.09$ \\
\hline $\mathrm{Na}(\mathrm{mmol} / \mathrm{L})$ & $144.0 \pm 1.7$ & $143.2 \pm 1.3$ & $144.4 \pm 1.4$ & $144.5 \pm 1.2$ \\
\hline $\begin{array}{l}\mathrm{K}(\mathrm{mmol} / \mathrm{L}) \\
\mathrm{Cl}(\mathrm{mmol} / \mathrm{L})\end{array}$ & $\begin{array}{r}4.4 \pm 0.3 \\
104.9 \pm 1.4\end{array}$ & $\begin{array}{r}4.5 \pm 0.3 \\
105.4 \pm 1.6\end{array}$ & $\begin{array}{r}4.7 \pm 0.2 \\
106.2 \pm 1.7\end{array}$ & $\begin{array}{c}4.6 \pm 0.3 \\
106.8 \pm 1.4^{*}\end{array}$ \\
\hline Recovery group & & & & \\
\hline No. of rats & 6 & - & - & 6 \\
\hline ALT (U/L) & $28.2 \pm 4.4$ & - & - & $28.2 \pm 7.4$ \\
\hline AST (U/L) & $119.6 \pm 53.1$ & - & - & $114.2 \pm 31.1$ \\
\hline ALP (U/L) & $72.3 \pm 17.3$ & - & - & $78.4 \pm 15.7$ \\
\hline Glu (mg/dL) & $147.7 \pm 9.0$ & - & - & $149.1 \pm 14.2$ \\
\hline BUN (mg/dL) & $10.74 \pm 1.09$ & - & - & $12.11 \pm 2.35$ \\
\hline Crea (mg/dL) & $0.30 \pm 0.02$ & - & - & $0.33 \pm 0.05$ \\
\hline T-Bil (mg/dL) & $0.22 \pm 0.04$ & - & - & $0.26 \pm 0.08$ \\
\hline T-Chol (mg/dL) & $95.45 \pm 9.66$ & - & - & $107.95 \pm 27.04$ \\
\hline TG (mg/dL) & $74.75 \pm 39.78$ & - & - & $77.16 \pm 36.78$ \\
\hline TP (g/dL) & $7.1 \pm 0.3$ & - & - & $6.9 \pm 0.4$ \\
\hline Alb (g/dL) & $4.3 \pm 0.3$ & - & - & $4.2 \pm 0.2$ \\
\hline A/G ratio & $1.56 \pm 0.18$ & - & - & $1.65 \pm 0.44$ \\
\hline$P(\mathrm{mg} / \mathrm{dL})$ & $6.48 \pm 0.34$ & - & - & $6.20 \pm 0.53$ \\
\hline $\mathrm{Ca}(\mathrm{mmol} / \mathrm{L})$ & $2.74 \pm 0.08$ & - & - & $2.71 \pm 0.05$ \\
\hline $\mathrm{Na}(\mathrm{mmol} / \mathrm{L})$ & $142.5 \pm 1.0$ & - & - & $142.5 \pm 0.8$ \\
\hline $\begin{array}{l}\mathrm{K}(\mathrm{mmol} / \mathrm{L}) \\
\mathrm{Cl}(\mathrm{mmol} / \mathrm{L})\end{array}$ & $\begin{array}{r}4.7 \pm 0.2 \\
101.8 \pm 1.5\end{array}$ & - & - & $\begin{array}{r}4.7 \pm 0.3 \\
102.3 \pm 1.9\end{array}$ \\
\hline
\end{tabular}

from ginseng leaves, when orally administered once daily to SD rats for a period of 13 weeks and to assess the reversibility of any effects after a 4-week recovery phase. The prospected clinical dose of UG0712 was 10 $\mathrm{mg} / \mathrm{kg}$ if human eats maximum $600 \mathrm{mg} / \mathrm{day} / 60 \mathrm{~kg}$. Through the results of 4-week dose range finding study, treatment-related alterations were noted in the blood chemistry data in $2,000 \mathrm{mg} / \mathrm{kg}$ treated animals, but systemic toxicity attributed to treatment was not recognized (data not shown). Therefore, the dose levels were established as $100 \mathrm{mg} / \mathrm{kg}$ (10 times of the prospected clinical dose), $400 \mathrm{mg} / \mathrm{kg}$, and 1,600 mg/kg with the geometric ratio of 4 in this study.

Under the conditions of our study, no treatment-related death was observed. Salivation was observed but this finding is considered to be the temporary sign due to the administration of test substance. Soft stool, diarrhea and mucous stool were occasionally observed in $1,600 \mathrm{mg} /$ $\mathrm{kg}$ treated groups. Excessive administration of ginsenoside can cause the symptoms because unabsorbed ginsenoside into gastrointestinal duct can be excreted [16,17]. The temporal significant increase in body weights and food consumptions were observed in female with $1,600 \mathrm{mg} /$ $\mathrm{kg}$, consistent with the previous study [18]. Opthalmological 
Table 3. Continued

\begin{tabular}{|c|c|c|c|c|}
\hline Parameters & Vehicle control & $100 \mathrm{mg} / \mathrm{kg}$ & $400 \mathrm{mg} / \mathrm{kg}$ & $1,600 \mathrm{mg} / \mathrm{kg}$ \\
\hline $\begin{array}{l}\text { Females } \\
\text { Main group }\end{array}$ & & & & \\
\hline No. of rats & 11 & 11 & 11 & 11 \\
\hline ALT (U/L) & $25.8 \pm 3.9$ & $22.8 \pm 5.7$ & $23.8 \pm 9.6$ & $25.0 \pm 3.5$ \\
\hline AST (U/L) & $94.5 \pm 19.8$ & $87.3 \pm 22.8$ & $81.5 \pm 21.7$ & $84.9 \pm 26.8$ \\
\hline ALP (U/L) & $49.7 \pm 13.5$ & $40.5 \pm 9.6$ & $41.7 \pm 13.7$ & $43.1 \pm 9.4$ \\
\hline Glu (mg/dL) & $121.3 \pm 10.1$ & $120.2 \pm 10.6$ & $120.6 \pm 15.9$ & $117.1 \pm 13.3$ \\
\hline BUN (mg/dL) & $12.05 \pm 1.04$ & $11.32 \pm 1.55$ & $11.02 \pm 1.90$ & $12.83 \pm 1.81$ \\
\hline Crea (mg/dL) & $0.36 \pm 0.06$ & $0.37 \pm 0.05$ & $0.36 \pm 0.04$ & $0.38 \pm 0.03$ \\
\hline T-Bil (mg/dL) & $0.14 \pm 0.03$ & $0.16 \pm 0.03$ & $0.18 \pm 0.08$ & $0.14 \pm 0.05$ \\
\hline T-Chol (mg/dL) & $74.56 \pm 21.12$ & $81.41 \pm 15.15$ & $91.14 \pm 19.02$ & $89.63 \pm 13.40$ \\
\hline TG (mg/dL) & $38.04 \pm 18.10$ & $44.00 \pm 21.04$ & $42.31 \pm 13.18$ & $40.62 \pm 16.28$ \\
\hline TP (g/dL) & $6.9 \pm 0.4$ & $7.2 \pm 0.6$ & $7.5 \pm 0.6^{*}$ & $7.5 \pm 0.7^{*}$ \\
\hline Alb (g/dL) & $4.8 \pm 0.3$ & $5.0 \pm 0.5$ & $5.3 \pm 0.5$ & $5.2 \pm 0.5$ \\
\hline A/G ratio & $2.38 \pm 0.48$ & $2.36 \pm 0.22$ & $2.42 \pm 0.24$ & $2.25 \pm 0.25$ \\
\hline$P(\mathrm{mg} / \mathrm{dL})$ & $6.54 \pm 0.75$ & $6.40 \pm 0.80$ & $6.14 \pm 0.81$ & $6.23 \pm 0.61$ \\
\hline $\mathrm{Ca}(\mathrm{mmol} / \mathrm{L})$ & $2.68 \pm 0.08$ & $2.72 \pm 0.08$ & $2.74 \pm 0.10$ & $2.75 \pm 0.08$ \\
\hline $\mathrm{Na}(\mathrm{mmol} / \mathrm{L})$ & $142.5 \pm 1.3$ & $143.5 \pm 1.5$ & $143.5 \pm 0.8$ & $142.6 \pm 1.7$ \\
\hline $\begin{array}{l}\mathrm{K}(\mathrm{mmol} / \mathrm{L}) \\
\mathrm{Cl}(\mathrm{mmol} / \mathrm{L})\end{array}$ & $\begin{array}{r}4.4 \pm 0.2 \\
105.7 \pm 1.8\end{array}$ & $\begin{array}{r}4.2 \pm 0.3 \\
105.7 \pm 2.0\end{array}$ & $\begin{array}{r}4.3 \pm 0.3 \\
105.5 \pm 1.6\end{array}$ & $\begin{array}{r}4.4 \pm 0.3 \\
105.1 \pm 1.6\end{array}$ \\
\hline $\begin{array}{l}\text { Recovery group } \\
\text { No. of rats }\end{array}$ & 6 & - & - & 6 \\
\hline ALT (U/L) & $27.0 \pm 5.6$ & - & - & $32.2 \pm 14.4$ \\
\hline AST (U/L) & $93.2 \pm 23.6$ & - & - & $107.5 \pm 24.1$ \\
\hline ALP (U/L) & $37.4 \pm 12.2$ & - & - & $30.1 \pm 7.4$ \\
\hline Glu (mg/dL) & $137.1 \pm 7.8$ & - & - & $127.4 \pm 3.9^{*}$ \\
\hline BUN (mg/dL) & $14.55 \pm 1.71$ & - & - & $14.03 \pm 2.43$ \\
\hline Crea (mg/dL) & $0.41 \pm 0.07$ & - & - & $0.39 \pm 0.05$ \\
\hline T-Bil (mg/dL) & $0.26 \pm 0.07$ & - & - & $0.22 \pm 0.02$ \\
\hline T-Chol (mg/dL) & $125.35 \pm 15.44$ & - & - & $100.52 \pm 26.96$ \\
\hline TG (mg/dL) & $40.81 \pm 7.48$ & - & - & $64.40 \pm 43.41$ \\
\hline $\mathrm{TP}(\mathrm{g} / \mathrm{dL})$ & $7.8 \pm 0.6$ & - & - & $7.8 \pm 0.5$ \\
\hline Alb (g/dL) & $5.4 \pm 0.4$ & - & - & $5.5 \pm 0.4$ \\
\hline A/G ratio & $2.39 \pm 0.37$ & - & - & $2.45 \pm 0.50$ \\
\hline$P(\mathrm{mg} / \mathrm{dL})$ & $5.07 \pm 0.79$ & - & - & $4.71 \pm 0.79$ \\
\hline $\mathrm{Ca}(\mathrm{mmol} / \mathrm{L})$ & $2.85 \pm 0.05$ & - & - & $2.84 \pm 0.10$ \\
\hline $\mathrm{Na}(\mathrm{mmol} / \mathrm{L})$ & $142.8 \pm 1.2$ & - & - & $143.2 \pm 0.8$ \\
\hline $\begin{array}{l}\mathrm{K}(\mathrm{mmol} / \mathrm{L}) \\
\mathrm{Cl}(\mathrm{mmol} / \mathrm{L})\end{array}$ & $\begin{array}{r}4.3 \pm 0.2 \\
101.8 \pm 2.8\end{array}$ & - & - & $\begin{array}{r}3.9 \pm 0.2^{*} \\
102.3 \pm 1.0\end{array}$ \\
\hline
\end{tabular}

Values are expressed as means \pm SD. ${ }^{*} P<0.05$ vs vehicle control.

examination failed to detect any abnormalities and urinalysis parameters were unaffected. The increase of prothrombin time and the decreases of platelet, reticulocyte and eosinophil were observed in the recovery group. However, since these changes could not be observed in the main group, they were not considered a toxicological result of UG0712 treatment.

Serum chemistry data showed that chloride $(\mathrm{Cl})$ and total protein (TP) were dose-dependently increased. Generally, the highest concentration of anion was contained in crude drugs and increased intake of $\mathrm{Cl}^{-}$via oral and intravenous may also lead to hyperchloremia
[19]. Yamamoto et al. (1978) had shown that protein synthesis was increased by ginsenoside. In addition, these values were within the normal range and no differences were found in a recovery period.

The absolute and relative liver weights were changed due to UG0712 treatment. However, there were no abnormalities at serum chemistry data, and histopathological changes also not identified in liver. It was also reported that Korean red ginseng accelerated the liver generation and ameliorated liver injury after hepatectomy in rats and dogs [20,21]. Therefore, the increase of liver weight was considered to be pharma- 
Table 4. Absolute and relative organ weight in male and female rats treated with UG0712

\begin{tabular}{|c|c|c|c|c|}
\hline Parameters & Vehicle control & $100 \mathrm{mg} / \mathrm{kg}$ & $400 \mathrm{mg} / \mathrm{kg}$ & $1,600 \mathrm{mg} / \mathrm{kg}$ \\
\hline $\begin{array}{l}\text { Males } \\
\text { Main group } \\
\text { No. of rats }\end{array}$ & 11 & 11 & 11 & 11 \\
\hline $\begin{array}{l}\text { Brain (g) } \\
\text { (g/BW \%) }\end{array}$ & $\begin{array}{l}2.02 \pm 0.05 \\
0.34 \pm 0.02\end{array}$ & $\begin{array}{l}2.06 \pm 0.08 \\
0.36 \pm 0.03\end{array}$ & $\begin{array}{l}2.05 \pm 0.05 \\
0.33 \pm 0.02\end{array}$ & $\begin{array}{l}2.04 \pm 0.07 \\
0.35 \pm 0.03\end{array}$ \\
\hline $\begin{array}{l}\text { Pituitary (g) } \\
\text { (g/BW \%) }\end{array}$ & $\begin{array}{l}0.0133 \pm 0.0007 \\
0.0022 \pm 0.0002\end{array}$ & $\begin{array}{l}0.0125 \pm 0.0016 \\
0.0022 \pm 0.0004\end{array}$ & $\begin{array}{l}0.0128 \pm 0.0017 \\
0.0021 \pm 0.0002\end{array}$ & $\begin{array}{l}0.0130 \pm 0.0017 \\
0.0022 \pm 0.0002\end{array}$ \\
\hline $\begin{array}{l}\text { Heart (g) } \\
(\mathrm{g} / \mathrm{BW} \%)\end{array}$ & $\begin{array}{l}1.58 \pm 0.10 \\
0.26 \pm 0.01\end{array}$ & $\begin{array}{l}1.62 \pm 0.11 \\
0.28 \pm 0.01\end{array}$ & $\begin{array}{l}1.61 \pm 0.10 \\
0.26 \pm 0.02\end{array}$ & $\begin{array}{l}1.57 \pm 0.12 \\
0.27 \pm 0.02\end{array}$ \\
\hline $\begin{array}{l}\text { Lung (g) } \\
\text { (g/BW \%) }\end{array}$ & $\begin{array}{l}1.67 \pm 0.13 \\
0.28 \pm 0.02\end{array}$ & $\begin{array}{l}1.67 \pm 0.12 \\
0.29 \pm 0.02\end{array}$ & $\begin{array}{l}1.73 \pm 0.11 \\
0.28 \pm 0.02\end{array}$ & $\begin{array}{l}1.71 \pm 0.15 \\
0.29 \pm 0.02\end{array}$ \\
\hline $\begin{array}{l}\text { Liver (g) } \\
\text { (g/BW \%) }\end{array}$ & $\begin{array}{r}17.15 \pm 1.59 \\
2.89 \pm 0.17\end{array}$ & $\begin{array}{r}16.58 \pm 2.40 \\
2.84 \pm 0.23\end{array}$ & $\begin{array}{r}18.05 \pm 1.53 \\
2.90 \pm 0.21\end{array}$ & $\begin{array}{r}16.71 \pm 1.63 \\
2.85 \pm 0.25\end{array}$ \\
\hline $\begin{array}{l}\text { Spleen (g) } \\
(\mathrm{g} / \mathrm{BW} \%)\end{array}$ & $\begin{array}{l}0.89 \pm 0.07 \\
0.15 \pm 0.01\end{array}$ & $\begin{array}{l}0.88 \pm 0.13 \\
0.15 \pm 0.02\end{array}$ & $\begin{array}{l}0.91 \pm 0.26 \\
0.15 \pm 0.04\end{array}$ & $\begin{array}{l}0.84 \pm 0.08 \\
0.14 \pm 0.02\end{array}$ \\
\hline $\begin{array}{l}\text { Left kidney (g) } \\
\text { (g/BW \%) } \\
\text { Right kidney (g) } \\
\text { (g/BW \%) }\end{array}$ & $\begin{array}{l}1.64 \pm 0.18 \\
0.27 \pm-.02 \\
1.63 \pm 0.17 \\
0.27 \pm 0.02\end{array}$ & $\begin{array}{l}1.57 \pm 0.14 \\
0.27 \pm 0.02 \\
1.64 \pm 0.17 \\
0.28 \pm 0.02\end{array}$ & $\begin{array}{l}1.64 \pm 0.18 \\
0.26 \pm 0.02 \\
1.66 \pm 0.14 \\
0.27 \pm 0.02\end{array}$ & $\begin{array}{l}1.60 \pm 0.15 \\
0.27 \pm 0.02 \\
1.61 \pm 0.13 \\
0.27 \pm 0.02\end{array}$ \\
\hline $\begin{array}{l}\text { Left adrenal (g) } \\
\text { (g/BW \%) } \\
\text { Right adrenal (g) } \\
\text { (g/BW \%) }\end{array}$ & $\begin{array}{l}0.0305 \pm 0.0042 \\
0.0051 \pm 0.0007 \\
0.0291 \pm 0.0046 \\
0.0049 \pm 0.0008\end{array}$ & $\begin{array}{l}0.0295 \pm 0.0024 \\
0.0051 \pm 0.0005 \\
0.0284 \pm 0.0024 \\
0.0049 \pm 0.0004\end{array}$ & $\begin{array}{l}0.0316 \pm 0.0047 \\
0.0051 \pm 0.0008 \\
0.0304 \pm 0.0037 \\
0.0049 \pm 0.0007\end{array}$ & $\begin{array}{l}0.0306 \pm 0.0044 \\
0.0053 \pm 0.0010 \\
0.0295 \pm 0.0024 \\
0.0051 \pm 0.0006\end{array}$ \\
\hline $\begin{array}{l}\text { Left testis (g) } \\
\text { (g/BW \%) } \\
\text { Right testis (g) } \\
\text { (g/BW \%) }\end{array}$ & $\begin{array}{l}1.69 \pm 0.12 \\
0.28 \pm 0.02 \\
1.69 \pm 0.15 \\
0.28 \pm 0.02\end{array}$ & $\begin{array}{l}1.69 \pm 0.12 \\
0.29 \pm 0.03 \\
1.70 \pm 0.12 \\
0.29 \pm 0.02\end{array}$ & $\begin{array}{l}1.60 \pm 0.12 \\
0.26 \pm 0.01^{*} \\
1.60 \pm 0.15 \\
0.26 \pm 0.01^{*}\end{array}$ & $\begin{array}{l}1.65 \pm 0.13 \\
0.28 \pm 0.03 \\
1.67 \pm 0.12 \\
0.29 \pm 0.03\end{array}$ \\
\hline Prostate (g) & $0.74 \pm 0.17$ & $0.62 \pm 0.09$ & $0.70 \pm 0.22$ & $0.70 \pm 0.16$ \\
\hline (g/BW \%) & $0.12 \pm 0.03$ & $0.11 \pm 0.02$ & $0.11 \pm 0.03$ & $0.12 \pm 0.03$ \\
\hline $\begin{array}{l}\text { Recovery group } \\
\text { No. of rats }\end{array}$ & 6 & 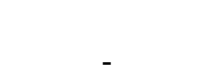 & 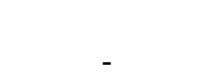 & 6 \\
\hline $\begin{array}{l}\text { Brain (g) } \\
\text { (g/BW \%) }\end{array}$ & $\begin{array}{l}2.10 \pm 0.09 \\
0.36 \pm 0.03\end{array}$ & - & - & $\begin{array}{l}2.08 \pm 0.08 \\
0.34 \pm 0.03\end{array}$ \\
\hline $\begin{array}{l}\text { Pituitary (g) } \\
\text { (g/BW \%) }\end{array}$ & $\begin{array}{l}0.0128 \pm 0.0007 \\
0.0022 \pm 0.0001\end{array}$ & - & - & $\begin{array}{l}0.0121 \pm 0.0015 \\
0.0019 \pm 0.0002^{*}\end{array}$ \\
\hline $\begin{array}{l}\text { Heart (g) } \\
\text { (g/BW \%) }\end{array}$ & $\begin{array}{l}1.57 \pm 0.15 \\
0.27 \pm 0.02\end{array}$ & - & - & $\begin{array}{l}1.66 \pm 0.14 \\
0.27 \pm 0.02\end{array}$ \\
\hline $\begin{array}{l}\text { Lung (g) } \\
\text { (g/BW \%) }\end{array}$ & $\begin{array}{l}1.76 \pm 0.14 \\
0.30 \pm 0.02\end{array}$ & - & - & $\begin{array}{l}1.76 \pm 0.08 \\
0.28 \pm 0.02\end{array}$ \\
\hline $\begin{array}{l}\text { Liver (g) } \\
\text { (g/BW \%) }\end{array}$ & $\begin{array}{r}15.37 \pm 1.67 \\
2.60 \pm 0.23\end{array}$ & - & - & $\begin{array}{r}15.89 \pm 1.05 \\
2.57 \pm 0.26\end{array}$ \\
\hline $\begin{array}{l}\text { Spleen (g) } \\
(\mathrm{g} / \mathrm{BW} \%)\end{array}$ & $\begin{array}{l}0.89 \pm 0.18 \\
0.15 \pm 0.03\end{array}$ & - & - & $\begin{array}{l}0.87 \pm 0.11 \\
0.14 \pm 0.02\end{array}$ \\
\hline $\begin{array}{l}\text { Left kidney (g) } \\
\text { (g/BW \%) } \\
\text { Right kidney (g) } \\
\text { (g/BW \%) }\end{array}$ & $\begin{array}{l}1.65 \pm 0.16 \\
0.28 \pm 0.01 \\
1.65 \pm 0.18 \\
0.28 \pm 0.02\end{array}$ & $\begin{array}{l}- \\
- \\
-\end{array}$ & $\begin{array}{l}- \\
- \\
-\end{array}$ & $\begin{array}{l}1.70 \pm 0.25 \\
0.27 \pm 0.04 \\
1.73 \pm 0.28 \\
0.28 \pm 0.04\end{array}$ \\
\hline $\begin{array}{l}\text { Left adrenal (g) } \\
\text { (g/BW \%) } \\
\text { Right adrenal (g) } \\
\text { (g/BW \%) }\end{array}$ & $\begin{array}{l}0.0279 \pm 0.0022 \\
0.0047 \pm 0.0004 \\
0.0263 \pm 0.0026 \\
0.0045 \pm 0.0005\end{array}$ & $\begin{array}{l}- \\
- \\
-\end{array}$ & $\begin{array}{l}- \\
- \\
-\end{array}$ & $\begin{array}{l}0.0300 \pm 0.0064 \\
0.0048 \pm 0.0011 \\
0.0288 \pm 0.0052 \\
0.0046 \pm 0.0008\end{array}$ \\
\hline $\begin{array}{l}\text { Left testis (g) } \\
\text { (g/BW \%) } \\
\text { Right testis (g) } \\
\text { (g/BW \%) }\end{array}$ & $\begin{array}{l}1.58 \pm 0.13 \\
0.27 \pm 0.02 \\
1.62 \pm 0.16 \\
0.27 \pm 0.03\end{array}$ & $\begin{array}{l}- \\
- \\
-\end{array}$ & $\begin{array}{l}- \\
- \\
-\end{array}$ & $\begin{array}{l}1.60 \pm 0.13 \\
0.26 \pm 0.03 \\
1.59 \pm 0.14 \\
0.26 \pm 0.03\end{array}$ \\
\hline Prostate (g) & $0.68 \pm 0.16$ & - & - & $0.79 \pm 0.16$ \\
\hline (g/BW \%) & $0.12 \pm 0.03$ & - & - & $0.13 \pm 0.02$ \\
\hline
\end{tabular}


Table 4. Continued

\begin{tabular}{|c|c|c|c|c|}
\hline Parameters & Vehicle control & $100 \mathrm{mg} / \mathrm{kg}$ & $400 \mathrm{mg} / \mathrm{kg}$ & $1600 \mathrm{mg} / \mathrm{kg}$ \\
\hline $\begin{array}{l}\text { Females } \\
\text { Main group }\end{array}$ & 11 & 11 & 11 & 11 \\
\hline $\begin{array}{l}\text { Brain (g) } \\
\text { (g/BW \%) }\end{array}$ & $\begin{array}{l}1.90 \pm 0.11 \\
0.64 \pm 0.09\end{array}$ & $\begin{array}{l}1.86 \pm 0.04 \\
0.60 \pm 0.05\end{array}$ & $\begin{array}{l}1.90 \pm 0.13 \\
0.60 \pm 0.05\end{array}$ & $\begin{array}{l}1.91 \pm 0.08 \\
0.61 \pm 0.05\end{array}$ \\
\hline $\begin{array}{l}\text { Pituitary (g) } \\
\text { (g/BW \%) }\end{array}$ & $\begin{array}{l}0.0161 \pm 0.0034 \\
0.0055 \pm 0.0016\end{array}$ & $\begin{array}{l}0.0170 \pm 0.0028 \\
0.0055 \pm 0.0011\end{array}$ & $\begin{array}{l}0.0180 \pm 0.0042 \\
0.0057 \pm 0.0014\end{array}$ & $\begin{array}{l}0.0173 \pm 0.0025 \\
0.0055 \pm 0.0009\end{array}$ \\
\hline $\begin{array}{l}\text { Heart (g) } \\
(\mathrm{g} / \mathrm{BW} \%)\end{array}$ & $\begin{array}{l}0.95 \pm 0.12 \\
0.32 \pm 0.03\end{array}$ & $\begin{array}{l}0.99 \pm 0.09 \\
0.32 \pm 0.03\end{array}$ & $\begin{array}{l}1.01 \pm 0.11 \\
0.32 \pm 0.02\end{array}$ & $\begin{array}{l}1.01 \pm 0.08 \\
0.32 \pm 0.02\end{array}$ \\
\hline $\begin{array}{l}\text { Lung (g) } \\
\text { (g/BW \%) }\end{array}$ & $\begin{array}{l}1.22 \pm 0.13 \\
0.41 \pm 0.03\end{array}$ & $\begin{array}{l}1.24 \pm 0.07 \\
0.40 \pm 0.03\end{array}$ & $\begin{array}{l}1.22 \pm 0.07 \\
0.39 \pm 0.03\end{array}$ & $\begin{array}{l}1.24 \pm 0.09 \\
0.40 \pm 0.03\end{array}$ \\
\hline $\begin{array}{l}\text { Liver (g) } \\
\text { (g/BW \%) }\end{array}$ & $\begin{array}{l}7.61 \pm 1.02 \\
2.53 \pm 0.10\end{array}$ & $\begin{array}{l}8.41 \pm 0.66 \\
2.69 \pm 0.12\end{array}$ & $\begin{array}{l}8.84 \pm 0.96^{* *} \\
2.77 \pm 0.23^{* *}\end{array}$ & $\begin{array}{l}8.85 \pm 0.85^{\star *} \\
2.83 \pm 0.20^{\star *}\end{array}$ \\
\hline $\begin{array}{l}\text { Spleen (g) } \\
\text { (g/BW \%) }\end{array}$ & $\begin{array}{l}0.52 \pm 0.08 \\
0.17 \pm 0.02\end{array}$ & $\begin{array}{l}0.54 \pm 0.07 \\
0.17 \pm 0.02\end{array}$ & $\begin{array}{l}0.51 \pm 0.05 \\
0.16 \pm 0.01\end{array}$ & $\begin{array}{l}0.51 \pm 0.05 \\
0.16 \pm 0.02\end{array}$ \\
\hline $\begin{array}{l}\text { Left kidney (g) } \\
\text { (g/BW \%) } \\
\text { Right kidney (g) } \\
\text { (g/BW \%) }\end{array}$ & $\begin{array}{l}0.85 \pm 0.09 \\
0.28 \pm 0.03 \\
0.90 \pm 0.12 \\
0.30 \pm 0.03\end{array}$ & $\begin{array}{l}0.88 \pm 0.07 \\
0.28 \pm 0.02 \\
0.89 \pm 0.06 \\
0.29 \pm 0.02\end{array}$ & $\begin{array}{l}0.91 \pm 0.10 \\
0.29 \pm 0.02 \\
0.94 \pm 0.09 \\
0.29 \pm 0.02\end{array}$ & $\begin{array}{l}0.90 \pm 0.05 \\
0.29 \pm 0.03 \\
0.92 \pm 0.06 \\
0.30 \pm 0.03\end{array}$ \\
\hline $\begin{array}{l}\text { Left adrenal (g) } \\
\text { (g/BW \%) } \\
\text { Right adrenal (g) } \\
\text { (g/BW \%) }\end{array}$ & $\begin{array}{l}0.0309 \pm 0.0035 \\
0.0104 \pm 0.0013 \\
0.0303 \pm 0.0030 \\
0.0102 \pm 0.0012\end{array}$ & $\begin{array}{l}0.0311 \pm 0.0036 \\
0.0100 \pm 0.0018 \\
0.0306 \pm 0.0037 \\
0.0099 \pm 0.0016\end{array}$ & $\begin{array}{l}0.0300 \pm 0.0048 \\
0.0094 \pm 0.0013 \\
0.0314 \pm 0.0046 \\
0.0099 \pm 0.0016\end{array}$ & $\begin{array}{l}0.0330 \pm 0.0051 \\
0.0106 \pm 0.0016 \\
0.0320 \pm 0.0042 \\
0.0103 \pm 0.0014\end{array}$ \\
\hline $\begin{array}{l}\text { Left ovary (g) } \\
\text { (g/BW \%) } \\
\text { Right ovary (g) } \\
\text { (g/BW \%) }\end{array}$ & $\begin{array}{l}0.0377 \pm 0.0030 \\
0.0128 \pm 0.0022 \\
0.0361 \pm 0.0042 \\
0.0121 \pm 0.0015\end{array}$ & $\begin{array}{l}0.0347 \pm 0.0089 \\
0.0110 \pm 0.0024 \\
0.0386 \pm 0.0087 \\
0.0124 \pm 0.0031\end{array}$ & $\begin{array}{l}0.0345 \pm 0.0079 \\
0.0107 \pm 0.0020 \\
0.0382 \pm 0.0093 \\
0.0119 \pm 0.0026\end{array}$ & $\begin{array}{l}0.0339 \pm 0.0068 \\
0.0109 \pm 0.0022 \\
0.0393 \pm 0.0078 \\
0.0126 \pm 0.0027\end{array}$ \\
\hline Uterus (g) & $0.65 \pm 0.29$ & $0.83 \pm 0.47$ & $0.71 \pm 0.25$ & $0.66 \pm 0.20$ \\
\hline (g/BW \%) & $0.22 \pm 0.09$ & $0.27 \pm 0.14$ & $0.22 \pm 0.07$ & $0.21 \pm 0.06$ \\
\hline $\begin{array}{l}\text { Recovery group } \\
\text { No. of rats }\end{array}$ & 6 & - & - & 6 \\
\hline $\begin{array}{l}\text { Brain (g) } \\
(\mathrm{g} / \mathrm{BW} \%)\end{array}$ & $\begin{array}{l}1.97 \pm 0.10 \\
0.64 \pm 0.04\end{array}$ & - & - & $\begin{array}{l}1.91 \pm 0.10 \\
0.62 \pm 0.09\end{array}$ \\
\hline $\begin{array}{l}\text { Pituitary (g) } \\
\text { (g/BW \%) }\end{array}$ & $\begin{array}{l}0.0187 \pm 0.0071 \\
0.0060 \pm 0.0020\end{array}$ & - & - & $\begin{array}{l}0.0179 \pm 0.0046 \\
0.0057 \pm 0.0011\end{array}$ \\
\hline $\begin{array}{l}\text { Heart (g) } \\
\text { (g/BW \%) }\end{array}$ & $\begin{array}{l}0.98 \pm 0.09 \\
0.32 \pm 0.02\end{array}$ & - & - & $\begin{array}{l}1.01 \pm 0.10 \\
0.32 \pm 0.02\end{array}$ \\
\hline $\begin{array}{l}\text { Lung (g) } \\
\text { (g/BW \%) }\end{array}$ & $\begin{array}{l}1.26 \pm 0.07 \\
0.41 \pm 0.01\end{array}$ & - & - & $\begin{array}{l}1.28 \pm 0.05 \\
0.41 \pm 0.06\end{array}$ \\
\hline $\begin{array}{l}\text { Liver (g) } \\
\text { (g/BW \%) }\end{array}$ & $\begin{array}{l}7.84 \pm 0.67 \\
2.54 \pm 0.16\end{array}$ & - & - & $\begin{array}{l}8.02 \pm .120 \\
2.55 \pm 0.13\end{array}$ \\
\hline $\begin{array}{l}\text { Spleen (g) } \\
(\mathrm{g} / \mathrm{BW} \%)\end{array}$ & $\begin{array}{l}0.48 \pm 0.04 \\
0.16 \pm 0.02\end{array}$ & - & - & $\begin{array}{l}0.52 \pm 0.05 \\
0.17 \pm 0.03\end{array}$ \\
\hline $\begin{array}{l}\text { Left kidney (g) } \\
\text { (g/BW \%) } \\
\text { Right kidney (g) } \\
\text { (g/BW \%) }\end{array}$ & $\begin{array}{l}0.91 \pm 0.05 \\
0.29 \pm 0.01 \\
0.94 \pm 0.03 \\
0.31 \pm 0.01\end{array}$ & $\begin{array}{l}- \\
- \\
-\end{array}$ & $\begin{array}{l}- \\
- \\
-\end{array}$ & $\begin{array}{l}0.90 \pm 0.04 \\
0.29 \pm 0.03 \\
0.96 \pm 0.04 \\
0.31 \pm 0.04\end{array}$ \\
\hline $\begin{array}{l}\text { Left adrenal (g) } \\
\text { (g/BW \%) } \\
\text { Right adrenal (g) } \\
\text { (g/BW \%) }\end{array}$ & $\begin{array}{l}0.0307 \pm 0.0054 \\
0.0099 \pm 0.0014 \\
0.0303 \pm 0.0058 \\
0.0098 \pm 0.0016\end{array}$ & $\begin{array}{l}- \\
- \\
-\end{array}$ & $\begin{array}{l}- \\
- \\
-\end{array}$ & $\begin{array}{l}0.0340 \pm 0.0032 \\
0.0110 \pm 0.0016 \\
0.0302 \pm 0.0027 \\
0.0098 \pm 0.0018\end{array}$ \\
\hline $\begin{array}{l}\text { Left ovary (g) } \\
\text { (g/BW \%) } \\
\text { Right ovary (g) } \\
\text { (g/BW \%) }\end{array}$ & $\begin{array}{l}0.0386 \pm 0.0119 \\
0.0125 \pm 0.0039 \\
0.0383 \pm 0.0068 \\
0.0124 \pm 0.0023\end{array}$ & $\begin{array}{l}- \\
- \\
-\end{array}$ & $\begin{array}{l}- \\
- \\
-\end{array}$ & $\begin{array}{l}0.0387 \pm 0.0087 \\
0.0124 \pm 0.0027 \\
0.0395 \pm 0.0087 \\
0.0127 \pm 0.0032\end{array}$ \\
\hline Uterus (g) & $0.84 \pm 0.31$ & - & - & $0.84 \pm 0.26$ \\
\hline (g/BW \%) & $0.27 \pm 0.10$ & - & - & $0.27 \pm 0.09$ \\
\hline
\end{tabular}

BW: body weight; Values are expressed as means \pm SD. ${ }^{*} P<0.05$ vs vehicle control. 
cological effect attributed to treatment with UG0712. Although the relative weights of testes and pituitary in males were increased, their absolute weights and histopathological changes were not observed. Thus, these changes were not considered as a UG0712-induced abnormality.

In conclusion, repeated oral dose administration of UG0712 to SD rats for 13 weeks did not cause any toxic effects in rats of either sex up to dose of $1,600 \mathrm{mg} / \mathrm{kg} /$ day. The NOAEL(no observed adverse effect level) was considered to be more than $1,600 \mathrm{mg} / \mathrm{kg}$.

\section{References}

1. Yun TK. Brief introduction of Panax ginseng C.A. Meyer. J Korean Med Sci 2001; 16 Supp 1: S3-5.

2. Kiefer D, Pantuso T. Panax ginseng. Am Fam Physician 2003; 68(8): 1539-1542.

3. Leung KW, Wong AS. Pharmacology of ginsenosides: a literature review. Chin Med 2010; 5: 20.

4. Kim HS, Lee EH, Ko SR, Choi KJ, Park JH, Im DS. Effects of ginsenosides $\mathrm{Rg} 3$ and $\mathrm{Rh} 2$ on the proliferation of prostate cancer cells. Arch Pharm Res 2004; 27(4): 429-435.

5. Kim DH, Chung JH, Yoon JS, Ha YM, Bae S, Lee EK, Jung KJ, Kim MS, Kim YJ, Kim MK, Chung HY. Ginsenoside Rd inhibits the expressions of iNOS and COX-2 by suppressing NF-êB in LPS-stimulated RAW264.7 cells and mouse liver. J Ginseng Res 2013; 37(1): 54-63.

6. Hu G, Wu Z, Yang F, Zhao H, Liu X, Deng Y, Shi M, Zhao G. Ginsenoside Rd blocks AIF mitochondrio-nuclear translocation and NF-êB nuclear accumulation by inhibiting poly(ADP-ribose) polymerase-1 after focal cerebral ischemia in rats. Neurol Sci 2013; 34(12): 2101-2106.

7. Tian J, Fu F, Geng M, Jiang Y, Yang J, Jiang W, Wang C, Liu K. Neuroprotective effect of 20(S)-ginsenoside $\operatorname{Rg} 3$ on cerebral ischemia in rats. Neurosci Lett 2005; 374(2): 92-97.

8. Tian J, Zhang S, Li G, Liu Z, Xu B. 20(S)-ginsenoside Rg3, a neuroprotective agent, inhibits mitochondrial permeability transition pores in rat brain. Phytother Res 2009; 23(4): 486-491.

9. Chai H, Do S, Kim J, Kim D, Sung S, Lee Y, Woo S, Kang J.
UG0712, a novel ginsenoside composition enhances the endurance exercise capacity and fatigue recovery. Lab Anim Res 2009; 25: 207-212.

10. Chan PC, Peckham JC, Malarkey DE, Kissling GE, Travlos GS, Fu PP. Two-year toxicity and carcinogenicity studies of Panax ginseng in Fischer 344 rats and B6C3F1 mice. Am J Chin Med 2011; 39(4): 779-788.

11. Liu JP, Lu D, Nicholson RC, Li PY, Wang F. Toxicity of a novel anti-tumor agent 20(S)-ginsenoside Rg3: a 26-week intramuscular repeated administration study in Beagle dogs. Food Chem Toxicol 2011; 49(8): 1718-1727.

12. Liu JP, Lu D, Nicholson RC, Zhao WJ, Li PY, Wang F. Toxicity of a novel anti-tumor agent 20(S)-ginsenoside Rg3: a 26-week intramuscular repeated administration study in rats. Food Chem Toxicol 2012; 50(10): 3388-3396.

13. Lu D, Liu J, Zhao W, Li P. Chronic toxicity of ginsenoside Re on Sprague-Dawley rats. J Ethnopharmacol 2012; 144(3): 656-663.

14. Wan JB, Yang FQ, Li SP, Wang YT, Cui XM. Chemical characteristics for different parts of Panax notoginseng using pressurized liquid extraction and HPLC-ELSD. J Pharm Biomed Anal 2006; 41(5): 1596-1601.

15. Wang CZ, Wu JA, McEntee E, Yuan CS. Saponins composition in American ginseng leaf and berry assayed by high-performance liquid chromatography. J Agric Food Chem 2006; 54(6): 22612266.

16. Gu Y, Wang GJ, Sun JG, Jia YW, Wang W, Xu MJ, Lv T, Zheng YT, Sai Y. Pharmacokinetic characterization of ginsenoside $\mathrm{Rh} 2$, an anticancer nutrient from ginseng, in rats and dogs. Food Chem Toxicol 2009; 47(9): 2257-2268.

17. Kim U, Park MH, Kim DH, Yoo HH. Metabolite profiling of ginsenoside Re in rat urine and faeces after oral administration. Food Chem 2013; 136(3-4): 1364-1369.

18. Aphale AA, Chhibba AD, Kumbhakarna NR, Mateenuddin M, Dahat SH. Subacute toxicity study of the combination of ginseng (Panax ginseng) and ashwagandha (Withania somnifera) in rats: a safety assessment. Indian J Physiol Pharmacol 1998; 42(2): 299302.

19. Yang KS, Kim YK, B PS. Studies on the anion contents in crude drugs. Kor. J. Pharmacogn 1989; 20(1), 64.

20. Kwon YS, Jang KH. The effect of Korean red ginseng on liver regeneration after 70\% hepatectomy in rats. J Vet Med Sci 2004; 66(2): 193-195.

21. Kwon YS, Jang KH, Jang IH. The effects of Korean red ginseng (ginseng radix rubra) on liver regeneration after partial hepatectomy in dogs. J Vet Sci 2003; 4(1): 83-91. 